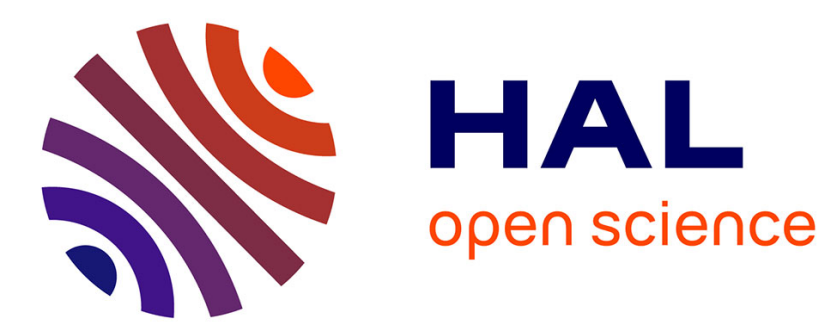

\title{
Transport pédiatrique médicalisé en France en 2018
}

\author{
G. Jourdain, N. Lodé, J.-L. Chabernaud
}

\section{To cite this version:}

G. Jourdain, N. Lodé, J.-L. Chabernaud. Transport pédiatrique médicalisé en France en 2018. Journal de Pédiatrie et de Puériculture, 2019, 32, pp.12 - 19. 10.1016/j.jpp.2018.10.005 . hal-03486197

\section{HAL Id: hal-03486197 \\ https://hal.science/hal-03486197}

Submitted on 20 Dec 2021

HAL is a multi-disciplinary open access archive for the deposit and dissemination of scientific research documents, whether they are published or not. The documents may come from teaching and research institutions in France or abroad, or from public or private research centers.
L'archive ouverte pluridisciplinaire HAL, est destinée au dépôt et à la diffusion de documents scientifiques de niveau recherche, publiés ou non, émanant des établissements d'enseignement et de recherche français ou étrangers, des laboratoires publics ou privés.

\section{(ㄷ)(1) $\$$}

Distributed under a Creative Commons Attribution - NonCommercial| 4.0 International 


\section{Transport pédiatrique médicalisé en France en 2018}

\section{Pediatric transfer by a retrieval team in France in 2018}

G. Jourdain $^{\text {a,b,* }}$, N. Lodéc ${ }^{c}$ J.-L. Chabernaud ${ }^{d}$

${ }^{a}$ SMUR 92 pédiatrique, 92141 Clamart cedex, France

${ }^{\text {b} C e n t r e ~ d e ~ R e ́ f e ́ r e n c e ~ M I N ~ 78-92-94, ~ s i t e ~ A ~ B e ́ c l e ̀ r e, ~ H o ̂ p i t a u x ~ U n i v e r s i t a i r e s ~ P a r i s ~ S u d ~}$ (APHP), 157, rue de la Porte-de-Trivaux, 92141 Clamart cedex, France

${ }^{\mathrm{c}}$ SMUR pédiatrique Robert Debré (SAMU de Paris), CHU Robert-Debré (AP-HP), 48 boulevard Sérurier, 75019 Paris, France

${ }^{\text {d} S M U R ~ p e ́ d i a t r i q u e ~ d e ~ C l a m a r t ~(S A M U ~ 92), ~ C H U ~ A n t o i n e-B e ́ c l e ̀ r e ~(A P-H P), ~ 157, ~ r u e ~ d e ~ l a ~}$ Porte-de-Trivaux, 92141 Clamart cedex, France

Correspondance : G. Jourdain, Centre de Référence MIN 78-92-94, site A Béclère, Hôpitaux Universitaires Paris Sud (APHP), 157, rue de la Porte-de-Trivaux, 92141 Clamart cedex, France

e-mail : gilles.jourdain@u-psud.fr

Tél : 0033145374614

Fax : 0033146324540 


\section{Résumé :}

Des équipes dédiées réalisent des transports médicalisés pédiatriques et néonatals depuis la fin des années 70 en France. Le GFRUP (Groupe Francophone de Réanimation et Urgences Pédiatriques) a réalisé un état des lieux en 2017 sur la situation en France métropolitaine. Il en ressort qu'il y a maintenant dans chacune des 12 régions métropolitaines au moins 2 équipes de SMUR pédiatrique. Le mode de fonctionnement et l'activité varient beaucoup d'une équipe à l'autre. Cela va de quelques centaines de transports à près de 2000. Les équipes réalisant le plus de transports sont en général dédiées, alors que les autres sont détachées «à la demande » de la réanimation à laquelle elles appartiennent. L'âge limite des enfants transportés varie aussi, certains SMUR ont une activité exclusivement néonatale et, d'autres transportent les enfants jusqu'à 18 ans.

Les SMUR pédiatriques évoluent en «s'appropriant » des techniques réservées jusqu'à présent aux services de réanimation, les exemples en sont la ventilation par oscillation à haute fréquence, l'hypothermie thérapeutique active, l'assistance circulatoire extracorporelle et l'échographie transthoracique.

La formation initiale et continue, médicale et paramédicale, fait partie des missions universitaires confiées aux SMUR pédiatriques.

La place des parents lors des soins est une problématique qui s'est imposée en réanimation et il en est de même pour les équipes de transport.

En presque un demi-siècle le rôle des SMUR pédiatriques a grandement évolué, de structure de sauvetage en dernier recours ils sont devenus un maillon rationnel de la chaîne de soins pédiatriques au sein des réseaux régionaux.

\section{Mots clés :}

Nouveau-né, enfant, transfert médicalisé, régionalisation, formation initiale 


\section{Summary:}

Dedicated teams perform neonatal and pediatric transports since the late 70 s in France. In 2017, GFRUP (Groupe Francophone de Réanimation et Urgences Pédiatriques) has ordered a survey about the French organization of pediatric retrieval teams. There are at least two teams in each of the 12 regions of metropolitan France. The amount of transfers varies widely from one team to another (a few hundreds to nearly 2000). Teams performing most transfers are dedicated while the other ones are "on demand". There is a great variation in the age limit: exclusive neonatal team for some and until 18 years for others. Pediatric retrieval teams are able to use sophisticated techniques which used to be usable only in intensive care unit like ventilation with high frequency oscillation, active therapeutic hypothermia, extracorporeal membrane oxygenation and trans-thoracic echocardiography.

Initial and continuing training are part of the duty of many university retrieval teams.

Family centered care is now a key point in transfers' management.

In half a century, neonatal and pediatric transport has widely evolved, and is now a recognized part of the healthcare chain inside perinatal and pediatric networks.

\section{Keywords:}

Newborn, child, retrieval team, regionalization, initial training 


\section{Introduction}

Les débuts du transport médicalisé pédiatrique remontent en France à la fin des années 70 [1]. Les textes réglementaires régissant ces transports ont progressivement été promulgués. En 2005 paraissait la circulaire de la Direction de l'hospitalisation et de l'organisation des soins (DHOS) relative à l'organisation du transport des nouveau-nés, nourrissons et enfants ${ }^{1}$. Elle considérait 3 niveaux de transports pédiatriques: ambulancier, infirmier et médicalisé. Nous ne traiterons que de ce dernier type dans cet article. Une enquête réalisée en 2011 sous l'égide du Groupe francophone de réanimation et urgences pédiatriques (GFRUP) [2] a fait un état des lieux des SMUR pédiatriques français. Sept ans plus tard il semble intéressant d'actualiser celui-ci et de développer plusieurs problématiques, nouvelles ou en expansion, tant au niveau technique, relationnel que de la formation.

\section{Etat des lieux en 2017}

Un questionnaire a été envoyé par mail à tous les responsables des SMUR pédiatriques référencés dans l'annuaire du GFRUP en septembre 2017 avec une relance par mail et par téléphone si nécessaire. Trente-deux réponses ont été obtenues. Ainsi en France, 30 villes possèdent au moins une antenne de SMUR pédiatrique. Deux villes bénéficient de 2 implantations distinctes, Paris avec 2 sites réalisant tous les types de transport et Nancy avec 2 SMUR, l'un exclusivement néonatal et l'autre pédiatrique, toutes les autres villes n'ont qu'une implantation. Dans l'étude de 2011, seulement 14 des 21 régions métropolitaines possédaient au moins une antenne de SMUR pédiatrique pour les transferts inter-hospitaliers pédiatriques [2]. La réforme de 2015 (avec effet au $1^{\text {er }}$ janvier 2016) de la structure géographique des régions a ramené ce nombre de 21 à 12 .

\footnotetext{
${ }^{1}$ Circulaire DHOS/01/2005/67 relative à l'organisation du transport des nouveau-nés, des nourrissons et des enfants.
} 
Chaque région métropolitaine possède maintenant au moins 2 antennes de SMUR pédiatrique. Huit régions disposent de 2 SMUR pédiatriques, une de 3 et 2 de 4 , avec un nombre maximum de 5 pour l'Ile de France (Tableau 1). Ceci a bien sûr un impact sur la rationalisation des transferts interhospitaliers, chaque ARS (Agence Régionale de Santé) de chaque région demandant une autonomie à ce niveau. Lorsqu'une des équipes d'une région est « engagée » sur un transfert, la seconde peut la suppléer en cas d'appels simultanés. Evidemment avec l'augmentation de taille des régions, qui peuvent aller jusqu'à $80000 \mathrm{~km}^{2}$, les délais d'intervention peuvent s'en trouver augmentés mais le principe de la régionalisation est renforcé. Le nombre d'habitants par région est probablement un déterminant, mais d'importance secondaire, sur le nombre d'antennes pédiatriques existant dans la région. Six régions ayant 5 millions d'habitants ou moins disposent de 2 équipes alors que 4 régions ayant 5,5 millions d'habitants ou plus disposent de 3, 4 ou 5 implantations. Cependant 2 régions comptant 6 millions d'habitants ne disposent que de 2 SMUR pédiatriques dans leur région.

Le nombre annuel d'interventions par équipe est certainement un marqueur plus représentatif des besoins par région. Quatre villes représentant 5 implantations réalisent plus de 1500 transports par an, 3 sont situées en Ile de France et la quatrième en Occitanie.

Une dizaine d'équipes comptent plus de 1000 interventions par an ; elles sont situées dans 6 régions différentes parmi les 7 plus peuplées de France métropolitaine. Sept équipes réalisent entre 500 et 1000 transports par an. Enfin 15 équipes réalisent moins de 500 transports par an (avec un minimum à 50 pour l'une d'elle).

La grande disparité dans le nombre annuel de transport influence la structure de fonctionnement. Les SMUR pédiatriques réalisant plus de 1000 transports par an ont tous une équipe dédiée H24 et pour ceux réalisant plus de 1500 transports une seconde 
en journée, 5 jours par semaine. Plus le nombre de transports diminue et moins il y a d'équipe dédiée. Dans ce cas le plus souvent du personnel médical et paramédical est pris sur le service de réanimation (néonatale ou pédiatrique) de rattachement dans la journée et il existe une astreinte pour la garde, les week-ends et les jours fériés. Il existe peu d'articles essayant d'évaluer la pertinence de ce système régionalisé [3]. Deux éléments clés ont été évalués par l'équipe italienne ayant publié cette étude : le coût unitaire par transport et le nombre de transports minimal par an afin que l'équipe conserve son efficacité. Ils ont conclu qu'un minimum de 300 transports par an permettait de valider ces 2 marqueurs. La grande majorité des équipes françaises répond à ces exigences.

Alors que dans certains pays européens où le transport est régionalisé ou orienté autour de bassins de population clairement identifiés le transport néonatal et le transport pédiatrique sont séparés c'est rarement le cas en France $[1,2,4]$. Seulement 5 équipes n'effectuent que des transferts néonatals et une seule équipe exclut ces transports pour ne réaliser que les transferts pédiatriques. Vingt-six équipes effectuent donc des transferts néonatals et pédiatriques, mais là aussi existe une hétérogénéité sur l'âge supérieur de prise en charge, allant de 2 ans jusqu'à plus de 15 ans (15/26 équipes se limitant à 7 ans ou moins), la raison le plus souvent invoquée est la contrainte matérielle tant au niveau des dimensions de la civière ou du module de transport que de la quantité et du volume des dispositifs médicaux.

Un autre point de différenciation est le type d'intervention réalisé. Dans la terminologie des SAMU-SMUR il existe 2 types de transferts : les «primaires » qui correspondent aux interventions dans des lieux non médicalisés (domicile, crèche, école...) et les «secondaires » ou transferts interhospitaliers. Une quinzaine d'équipes réalisent moins de $1 \%$ de leur activité en primaire alors qu'une dépasse les $40 \%$. 
Enfin pour terminer cet état des lieux nous allons voir que le personnel médical, toujours rompu à l'urgence, peut varier au niveau de sa spécialité. Dix-huit équipes n'ont recours qu'à des pédiatres, cinq intègrent des anesthésistes et neuf des médecins urgentistes. Le pourcentage allant de moins de $10 \%$ à plus de $75 \%$ pour 2 équipes du temps médical nécessaire à faire fonctionner la structure avec des «non pédiatres »

\section{Formation des équipes}

La quasi-totalité des antennes SMUR pédiatriques sont au sein d'hôpitaux universitaires ou dépendent de services universitaires. Cependant la présence d'un DES de pédiatrie est très étroitement liée au niveau d'activité du SMUR pédiatrique. Les dix équipes réalisant plus de 1000 transports par an, à l'exception de 2, ont un DES de pédiatrie à «temps plein», une faisant le choix de faire un roulement avec les internes de réanimation néonatale ou pédiatrique, la durée de présence au SMUR pouvant dans ces cas-là être d'un ou deux mois, l'autre n'en ayant pas. Deux équipes, parmi celles réalisant moins de 1000 transports ont un DES également à plein temps, six autres ont choisi de mutualiser leur interne avec la réanimation dont ils dépendent avec une durée de présence variable au sein du SMUR pédiatrique. Ainsi chaque année une vingtaine d'internes en pédiatrie effectue un semestre complet au sein d'un SMUR pédiatrique et un nombre équivalent a une vue plus superficielle du transport pédiatrique. Avec la création récente du DES d'urgences, la question d'accueillir en plus un certain nombre de ceux-ci va se poser, or le nombre de terrain de stage ne sera pas extensible.

À ceci s'ajoutent, en formation initiale, les élèves sages-femmes et les étudiants en soins infirmiers, il s'agit dans ce cas de stages optionnels qui ne couvrent qu'une partie variable de l'année. 
Enfin il existe une forte demande en formation continue, essentiellement pour des médecins. Le plus souvent ce sont des médecins exerçant en SMUR adulte et souhaitant approfondir leurs connaissances pédiatriques par le biais de diplômes universitaires.

En parallèle de cette formation pratique, l'ensemble des SMUR pédiatriques métropolitains réfléchit à la mise en place d'un enseignement théorique uniformisé sur les particularités du transport néonatal et pédiatrique qui serait accessible en ligne pour les DES de pédiatrie durant leur stage au sein d'un SMUR pédiatrique.

\section{Apport des nouvelles techniques}

De tout temps les techniques appliquées dans les services de réanimation ont ensuite été exportées vers les équipes de transport, avec des adaptations en rapport avec les contraintes spatiales et cinétiques. Les développements les plus récents concernent la ventilation de type oscillation à haute fréquence $(\mathrm{OHF})$, l'hypothermie thérapeutique active chez le nouveau-né présentant une encéphalopathie anoxo-ischémique, les transports avec une unité médicale d'assistance circulatoire (UMAC) et l'échographie transthoracique principalement à visée hémodynamique ainsi que les moyens non invasifs d'évaluation de l'hémodynamique.

Certaines équipes pratiquaient déjà l'OHF en transport depuis le milieu des années 90 [5], mais le matériel était totalement inadapté à l'installation dans une UMH, tant pour des raisons de volume que de consommation de fluides. Le nombre de transports ainsi réalisés chaque année était extrêmement faible. L'apparition de ventilateurs compacts et sobres en consommation de fluides tel le Fabian iOHF $^{\circledR}$ (Acutronic Medical System, Zurich, Switzerland) ont permis de réaliser des transports dans des conditions confortables pour des nouveau-nés considérés auparavant comme intransportables. Les principales indications concernent les nouveau-nés présentant une hypoxie réfractaire en ventilation conventionnelle et devant être transférés en vue d'une assistance respiratoire 
extracorporelle, ainsi que les prématurés avec instabilité hémodynamique (en cas de sepsis ou d'entérocolite). À l'échelle d'une région comme l'Ile de France cela représente, maintenant, annuellement entre 100 et 150 transports.

L'hypothermie thérapeutique active est maintenant reconnue pour améliorer le pronostic neurologique des nouveau-nés à terme ayant présenté une anoxie périnatale modérée à sévère, mais pour avoir le maximum d'efficacité elle doit être débutée le plus précocement possible et toujours avant la $6^{\mathrm{e}}$ heure de vie. Elle ne se pratique que dans des centres périnatals de type 3 . Il peut donc être nécessaire de transférer un nouveau-né d'un centre ne pratiquant pas cette technique vers un centre la pratiquant. Jusqu'à récemment les équipes de transport en France pratiquait une mise en hypothermie passive avec de grandes difficultés à atteindre ou maintenir la température cible voulue [6]. En accord avec le service récepteur le SMUR pédiatrique peut débuter cette thérapeutique active avant l'arrivée en réanimation et gagner un temps précieux grâce à un refroidissement rapide et précis. Cependant de nombreuses complications peuvent advenir avec cette technique et elle doit être réservée à des équipes entraînées à son maniement.

Les UMAC permettent le transport d'enfants en défaillance polyviscérale autrefois considérés intransportables, parfois sur plusieurs centaines de kilomètres. Le but est d'aller implanter une circulation extra-corporelle (CEC) afin de suppléer un poumon et/ou un cœur défaillant. Le type de CEC est veino-veineuse (prédominance pulmonaire) ou veino-artérielle (défaillance cardiaque prédominante ou associée). Les voies d'implantation des canules sont chirurgicales : jugulo-carotidienne chez le nouveau-né et le nourrisson, jugulo-fémorale après 2 ans et fémoro-fémorale, comme l'adulte, chez le grand enfant. Il faut avoir à disposition des canules et des circuits de tailles différentes. Une régulation thermique (réchauffement du circuit de CEC et des 
gaz) est indispensable chez ces enfants. La logistique est très importante dans ce type de transport (rôle de l'infirmier). L'ambulancier, spécifiquement formé à cette tâche, peut s'avérer complémentaire des équipes. Deux équipes de SMUR pédiatrique ont d'emblée participé au développement des UMAC pédiatriques. À Paris, celle de l'hôpital RobertDebré avec l'équipe médico-chirurgicale de l'hôpital Trousseau [7] et celle du CHU de Nantes. La particularité de l'équipe parisienne est d'être supra-régionale (Ile-de-France, nord et nord-ouest de la France) et de répartir les patients sur plusieurs centres d'ECMO (Extra Corporeal Membrane Oxygenation) : à Paris l'hôpital Trousseau et l'hôpital Necker plus rarement le Centre Chirurgical Marie Lannelongue (PlessisRobinson).

Les indications sont posées par le réanimateur du centre d'ECMO sur des critères précis demandés au centre requérant [7]. Chez le nouveau-né : syndrome d'inhalation méconiale, choc septique et hernie diaphragmatique prédominent, chez l'enfant: Syndrome de détresse respiratoire aigu et choc septique puis myocardite et parfois la mucoviscidose en prégreffe.

Pour améliorer la prise en charge et le transport de ces patients, des formations locales inter-équipes ont été mises en place et sont régulièrement suivies.

Comme pour l'expérience des centres d'ECMO [8], le nombre de transports UMAC effectués par équipe est un gage de sécurité [9]. L'équipe parisienne a effectué 19 transferts en UMAC en 2017 et la demande est croissante.

Enfin l'échographie transthoracique est appelée à se développer, probablement avec d'autres systèmes de monitorage non invasifs de l'hémodynamique telle la cardiométrie électrique avec l'ICON ${ }^{\circledR}$ (Osypka medical, Cardiotronic inc., La Jolla (CA), USA) [10]. La cardiométrie électrique repose sur la bioimpédance et a d'abord été appliquée dans la surveillance médicale des astronautes lors de la conquête spatiale, c'est une technologie 
totalement non invasive. Ces 2 techniques complémentaires permettent une évaluation précise de l'hémodynamique centrale des patients transportés, l'échographie de manière ponctuelle mais pouvant être répétée, la cardiométrie électrique de manière continue. L'échographie nécessite un apprentissage plus ou moins long suivant le niveau d'expertise recherché, la cardiométrie quasiment aucun. L'association de ces techniques permettra probablement de décider de pratiquer des remplissages vasculaires et initier des traitements avec des amines vasopressives plus rapidement et avec des arguments dépassant la clinique.

\section{Présence parentale}

Il y a une trentaine d'années la présence des parents lors de la prise en charge de leur nouveau-né ou enfant était, au mieux, tolérée ; actuellement il existe une forte demande, individuelle ou venant de groupes de parents, d'intégration au projet de soin de leur enfant. Il existe une abondante littérature sur le sujet et une synthèse récente a fait le point sur ce qui pouvait être proposé [11]. Il en ressort que la présence parentale durant les soins y compris très invasifs ainsi que pendant les manœuvres de réanimation consécutives à un arrêt cardiaque était bénéfique à long terme (6 mois) sur leur état psychologique. Cependant il existe très peu d'études de qualité pour étayer cette pratique et leur niveau de preuve est très faible. Pour la présence d'au moins un des deux parents durant le transfert dans l'ambulance de réanimation, il y a encore peu d'éléments publiés [12, 13]. En pratique, en France, l'attitude est extrêmement variable [14]. Certaines équipes n'acceptent pas de prendre les parents pendant les trajets alors que d'autres le proposent systématiquement. Un travail de réflexion et d'évaluation est absolument nécessaire à mener. S'il n'y a aucune raison de refuser de transporter les parents d'un nourrisson présentant une bronchiolite sévère, la question du transport de la mère avec son nouveau-né lorsqu'elle vient d'accoucher est difficile à trancher. La 
réponse appartient bien sûr à l'obstétricien, mais quel intervalle entre l'accouchement et le transfert peut-il être considéré comme raisonnable (au Royaume Uni il n'est pas exceptionnel que la sortie maternelle s'opère vers H6 post accouchement). Dans les cas de transfert très précoce après la naissance le père peut accompagner et ainsi donner des nouvelles à la mère en temps réel. C'est une solution qui semble apporter une certaine sérénité mais qui devrait également être évaluée.

\section{Points clés de l'évolution des SMUR pédiatriques depuis leur création}

Dans les années 1960, grossesse et accouchement ne bénéficiaient d'aucune collaboration obstétrico-pédiatrique et quelques transferts de nouveau-nés en grande détresse, étaient effectués plusieurs heures après leur naissance dans des incubateurs rudimentaires vers des services de pédiatrie générale. Dès 1969, l'équipe du SAMU de Paris faisait part de son expérience en matière de transport des nouveau-nés et nourrissons en détresse cardio-circulatoire [15]. En France, les deux premiers services de réanimation prenant en charge des nouveau-nés ont été, à Paris, l'unité de réanimation pédiatrique créée en novembre 1964 par le Pr Gilbert Huault à l'hôpital Saint-Vincent-de-Paul puis la première unité spécifiquement néonatale ouverte en 19661967 par le Pr Alexandre Minkowski au sein de la maternité de Port-Royal. À cette époque, les créateurs de la réanimation néonatale et pédiatrique en France affirment les risques du transfert en période néonatale et énoncent les grands principes auxquels il doit satisfaire, inventant la notion de «chaîne » (chaud, oxygène, sucre et asepsie) [16]. Lors de leur création au début des années 70, les anesthésistes-réanimateurs des équipes des Services Mobiles d'Urgence et de Réanimation (SMUR), du fait d'une expérience limitée des particularités physiologiques du nouveau-né et du nourrisson ainsi que de la spécificité des pathologies observées chez l'enfant avant l'âge de 5 ans, rencontraient d'importantes difficultés techniques de prise en charge [17]. 


\section{L'ère des pionniers}

Les SMUR pédiatriques ont été ainsi créés dans certaines régions à partir de 1976 sous l'impulsion des néonatologistes pour organiser et réaliser les transferts médicalisés en cas de détresse grave. Leurs interventions ont progressivement, et de plus en plus, touché des enfants de tous âges. En Ile-de-France, un dispositif régional composé de 5 équipes s'est constitué, d'abord à partir de 1976 dans les départements de Seine-StDenis (Dr Jean-Pierre Fournet, Montreuil) et des Hauts-de-Seine (Prs Jean-Claude Gabilan et Michel Dehan Antoine Béclère, Clamart), puis en 1980 dans Paris (Necker et Robert Debré) et le Val d'Oise (Pontoise et Argenteuil). Chacune de ces équipes, incluse dans l'un des SAMU départementaux d'Ile-de-France, est rattachée à une unité de réanimation néonatale ou pédiatrique d'un centre périnatal de type III. Une commission transport néonatal existe au sein de la Société Française de Néonatalogie (SFN) depuis 1999. Depuis une dizaine d'années, un groupe «SMUR pédiatriques » a été mis en place au sein du GFRUP. Les échanges ont lieu sous forme de réunions téléphoniques. Une journée de rencontre nationale a également lieu chaque année dans l'une de grandes villes universitaires d'implantation.

\section{Organisation régionale en Ile-de-France}

Dès 1980, les responsables médicaux des SMUR pédiatriques de l'Ile de France (au sein du Groupe d'Etudes en Néonatalogie et Urgences Pédiatriques de la région parisienne GENEUP-RP) décidèrent de travailler en commun pour améliorer la rapidité d'intervention auprès des enfants gravement malades et uniformiser leur prise en charge. L'inter-SMUR pédiatrique de l'Ile de France regroupant les SMUR pédiatriques des départements 75, 92, 93, 94 et 95 (seul SMUR ne faisant pas partie de l'AP-HP) fut ainsi mis sur pied [18].

Un répertoire de régulation commun fut établi définissant les différents secteurs 
d'intervention de chaque SMUR et la «rebascule» automatique de l'appel vers le SMUR pédiatrique le plus proche du lieu d'intervention, en cas d'indisponibilité du SMUR du secteur. Une feuille de transport commune à toute la région a également permis rapidement un codage informatique uniformisé.

Les particularités du dispositif francilien sont détaillées dans l'encadré 1

\section{Dans les autres régions françaises}

En 2011, il existait une équipe spécialisée de transport néonatal et/ou pédiatrique dans 14 régions françaises sur 21 et 7 régions disposaient de plusieurs équipes, soit au total 25 équipes (24 en CHU) [2]. En revanche, il n'existait aucune équipe spécialisée dans les départements d'outre-mer.

\section{Comparaison de l'organisation des transferts périnatals à l'échelle européenne}

En 1999, sous la direction du Pr Emile Papiernik, l'European Network for Perinatal Transport (EUROPET), action concertée d'évaluation et de recherche (dans laquelle la région Ile de France était représentée) sur les politiques de transferts périnatals (femme enceinte à risque et nouveau-né) dans les pays d'Europe, a établi les bases et les principes de l'organisation, de la formation des personnels et des matériels indispensables [19-21].

\section{Evolution de la réglementation des transferts pédiatriques}

Jusqu'en 1999, les équipes des SMUR pédiatriques ne comprenaient qu'un pédiatre réanimateur (souvent formé par le CES de pédiatrie) et un conducteur-ambulancier accompagnés parfois qu'un externe en pédiatrie ou un stagiaire (élève sage-femme, étudiant de spécialité en pédiatrie). Après la publication du décret sur les SMUR de 1997, obligeant toute équipe de SMUR à disposer d'un personnel infirmier, les SMUR pédiatriques purent créer des postes d'infirmières spécialisées (en réanimation néonatale ou pédiatrique) et/ou d'infirmières-puéricultrices parfois d'IADE, alors que les externes 
et les stagiaires en pédiatrie devenaient plus rares [22]. Deux événements ont été particulièrement décisifs : d'une part la parution de l'Article R-712-74-1 au sein du décret SAMU-SMUR de 1997, reconnaissant la possibilité de créer un SMUR pédiatrique dans un centre périnatal disposant d'une réanimation néonatale ou pédiatrique et d'autre part la publication (octobre 1998) des décrets d'application du Plan périnatalité 1994 puis celle du deuxième Plan périnatalité (2005-2007) permettant de développer (avec un budget spécifique) les transferts maternels et néonatals (médicalisés et paramédicalisés), grâce à la parution en 2005 de la circulaire de la DHOS sur le transport pédiatrique définissant, deux niveaux de soins et de surveillance: médicalisé et infirmier ${ }^{23},{ }^{45}$ Cela a permis la création d'équipes paramédicalisées (hospitalières ou privées) dans plusieurs régions. Dans les années 90 des postes formateurs de DES de pédiatrie dédiés aux SMUR pédiatriques furent également créés.

\section{Conclusion}

Le développement des SMUR pédiatriques en France a rapidement eu comme impact principal une amélioration considérable de la prise en charge des nouveau-nés, notamment des prématurés, en contribuant à la mise en place de la régionalisation des soins périnatals, mais également des nourrissons et des jeunes enfants [20,23].

Le sarcophage ou coque isotherme utilisé pendant des années par les différents SMUR pour maintenir la température des nouveau-nés a été remplacé dans les années 80 par

\footnotetext{
${ }^{2}$ Circulaire DHOS/01/2005/67 relative à l'organisation du transport des nouveau-nés, des nourrissons et des enfants.

${ }^{3}$ Décret $n^{\circ}$ 97-620 du 30 mai 1997 relatif aux conditions techniques de fonctionnement auxquelles doivent satisfaire les établissements de santé pour être autorisés à mettre en œuvre des services mobiles d'urgence et de réanimation.

${ }^{4}$ Ministère de l'emploi et de la solidarité, Décrets N08-899 et 98-9000 du 9 octobre 1998. Journal officiel de la République française, p 15343

${ }^{5}$ Plan Périnatalité 2005-2007 : Humanité, Proximité Sécurité Qualité
} 
des incubateurs de transport radiants très efficaces disposé sur un module compact rassemblant tous les appareils indispensables [1,15].

Les SMUR pédiatriques ont participé à l'amélioration des résultats obtenus en matière de santé périnatale et des pathologies urgentes de l'enfant, en permettant la diffusion de nombreuses techniques innovantes (monitorage hémodynamique et gazométrique transcutané, ventilation non invasive chez le nouveau-né et le nourrisson, ventilation mécanique synchronisée, surfactant exogène, ventilation à haute fréquence par oscillation, confort sédation-analgésie procédurale, hypothermie thérapeutique dans l'anoxie néonatale...), de protocoles de soins, ainsi que le champ de la prévention (MIN et accidents chez l'enfant) et le domaine de l'éthique et des soins palliatifs pédiatriques. Les responsables médicaux des SMUR pédiatriques sont par ailleurs rapidement devenus des interlocuteurs incontournables et écoutés des tutelles régionales et nationales (ARS, ministères...).

Conflits d'intérêts : aucun 


\section{Références}

1. Chabernaud JL, Ayachi A, Lode N, Lelong-Tissier MC, Diependaele JF, Menthonnex E. Histoire du transport néonatal : Progrès dans l'organisation au cours des 30 dernières années. Rev Med Perinat 2010;2:63-71

2. Naud J, Chabernaud JL. Organisation et perspectives des SMUR pédiatriques en France-Résultats de l'enquête du GFRUP. Réanimation 2011 ;20: 525-9

3. Bellini C, Pasquarella M, Ramenghi LA, Ambrosino D, Sciomachen AF. Evaluation of neonatal transport in a European country shows that regional provision is not cost-effective or sustainable and needs to be re-organised. Acta Paediatr. $2011 ; 107:$ 57-62.

4. Daussac E, Leslie A, Roth-Kleiner M, Zwissig M, Aufieri R, Gente M, et al. Le transfert néonatal par un SMUR pédiatrique, de la salle de naissance à la réanimation, en France... et ailleurs. Rev. Med. Périnat 2018;10:50-8

5. Jones P, Dauger S, Leger PL, Kessous K, Casadevall I, Maury I, et al. Mortality in children with respiratory failure transported using high-frequency oscillatory ventilation. Intensive Care Med. 2015;41:1363-4

6. Sellam A, Lode N, Ayachi A, Jourdain G, Chabernaud JL, Dauger S, et al. Passive hypothermia $\left(\geq 35-<36^{\circ} \mathrm{C}\right)$ during transport of newborns with hypoxicischaemic encephalopathy. PLoS ONE 2017;12: e0179068.

7. Rambaud J, Léger PL, Larroquet M, Amblard A, Lodé N, Guilbert J, et al. Transportation of children on extracorporeal membrane oxygenation : one year experience of the first neonatal and paediatric mobile ECMO team in the north of France. Intensive Care Med 2016 ;42:940-1 
8. Karamlou T, Vafaeezadeh M, Parrish AM, Cohen GA, Welke KF, Permut L, et al. Increased extracorporeal membrane oxygenation center case volume is associated with improved extracorporeal membrane oxygenation survival among pediatric patients. J Thorac Cardiovasc Surg 2013; 145:470-5

9. Niziolek KC, Preston TJ, Osborn EC. Transport While on Extracorporeal Membrane Oxygenation Support Crit Care Clin 2017; 33:883-96

10. Boët A, Jourdain G, Demontoux S, Hascoët S, Tissieres P, Rucker-Martin C, et al. Basic Hemodynamic Monitoring Using Ultrasound or Electrical Cardiometry During Transportation of Neonates and Infants. Pediatr Crit Care Med. 2017 ;18: e488-e493.

11. Davidson JE, Aslakson RA, Long AC, Puntillo, KA, Kross, EK, Hart J et al. Guidelines for Family-Centered Care in the Neonatal, Pediatric, and Adult ICU. Crit Care Med 2017; 45: 103-28.

12. Davies J, Tibby SM, Murdoch IA (2005) Should parents accompany critically ill children during inter-hospital transport? Arch Dis Child 2005 ; 90: 1270-3

13. Tasker RC. Inter-hospital transport for children and their parent(s). Arch Dis Child 2005; $90: 1217-8$

14. Gonzalez P, Jourdain G, Mehdi N, Douchin JL, Chabernaud JL (2008) Transfert néonatal en SMUR pédiatrique : évaluation de la relation avec les parents. Arch Pediatr 2008; 15: 976.

15. Hurtaud JP, Caille C, Ivanoff S, Martinez-Almoyna M. Transport médical des enfants en état grave. Revue du Praticien 1969; 19: 150-8

16. Huault G, Dehan M, Lejeune JA. Le transport du nouveau-né et du prématuré. Gazette Med de France 1972; 79: 6805-6821 
17. Cara M, Hurtaud JP, Ivanoff S, Rosemberg S, Caille C. Problèmes posés par le transport des nouveau-nés. Ann Anesth Franc 1972; 13: 111-118

18. Barbier ML, Chabernaud JL, Lavaud J, Février YM, Johanet S. Les transports médicalisés urgents en pédiatrie dans la région Ile de France. Arch Pediatr 1987; 44: $413-7$

19. Debauche C, Van Reempts P, Chabernaud JL, Kollee LAA, Zeitlin J. Maternal and neonatal transfer policies in Europe. Prenat Neonatal Med 1999; 4: 5-14

20. Sedin G, Agostino R, Chabernaud JL, Fenton AC, Kollee LAA. Technical aspects of neonatal transport in Europe. Prenat Neonatal Med 1999; 4: 35-45

21. Papiernik E, Zeitlin J, Chabernaud JL et les participants du projet EUROPET. L'Organisation des soins périnatals en Europe en 1996. In L'action collective périnatale en Ile de France (1996-2000) sous la direction d'Annie Serfaty et Emile Papiernik. Editions de l'Ecole Nationale de Santé Publique, Rennes, 2005. p 123-132.

22. Chabernaud JL. Analyse et point de vue des SMUR pédiatriques (Conférence de consensus sur la prise en charge de la femme enceinte, de l'accouchement et du nouveau-né). J Gynecol Obstet Biol Reprod 1998; 27 (suppl 2): 176-81.

23. Chabernaud JL. Analyse et point de vue des SMUR pédiatriques (Conférence de consensus sur la prise en charge de la femme enceinte, de l'accouchement et du nouveau-né). J Gynecol Obstet Biol Reprod 1998; 27 (2 suppl): 176-81 
Tableau 1 : Les équipes de SMUR pédiatrique dans les régions françaises

\begin{tabular}{|c|c|c|c|c|}
\hline Ville & Région & Nb Sites & $\begin{array}{l}\text { Habitants } \\
\text { en millions }\end{array}$ & $\begin{array}{c}\text { Nb transp } \\
>\text { à }\end{array}$ \\
\hline Lille & Hauts de France & 1 & \multirow{2}{*}{6} & 1000 \\
\hline Amiens & Hauts de France & 1 & & 1000 \\
\hline Rouen & Normandie & 1 & \multirow{2}{*}{3} & \\
\hline Caen & Normandie & 1 & & \\
\hline Rennes & Bretagne & 1 & \multirow{2}{*}{3} & \\
\hline Brest & Bretagne & 1 & & \\
\hline Nantes & Pays de la Loire & 1 & \multirow{2}{*}{4} & \\
\hline Angers & Pays de la Loire & 1 & & \\
\hline Tours & Centre Val de Loire & 1 & \multirow{2}{*}{2,5} & \\
\hline Orléans & Centre Val de Loire & 1 & & \\
\hline Poitiers & Nouvelle Aquitaine & 1 & \multirow{3}{*}{6} & \\
\hline Limoges & Nouvelle Aquitaine & 1 & & \\
\hline Bordeaux & Nouvelle Aquitaine & 1 & & 1000 \\
\hline Reims & Grand Est & 1 & \multirow{3}{*}{5,5} & \\
\hline Nancy & Grand Est & 2 & & \\
\hline Strasbourg & Grand Est & 1 & & \\
\hline Dijon & Bourgogne Franche Comté & 1 & \multirow{2}{*}{3} & \\
\hline Besançon & Bourgogne Franche Comté & 1 & & \\
\hline Lyon & Auvergne Rhône Alpes & 1 & \multirow{4}{*}{8} & 1000 \\
\hline Chambéry & Auvergne Rhône Alpes & 1 & & \\
\hline St Etienne & Auvergne Rhône Alpes & 1 & & \\
\hline Grenoble & Auvergne Rhône Alpes & 1 & & \\
\hline Nice & Provence Alpes Côte d'Azur & 1 & \multirow{2}{*}{5} & \\
\hline Marseille & Provence Alpes Côte d'Azur & 1 & & 1000 \\
\hline Montpellier & Occitanie & 1 & \multirow{2}{*}{6} & \\
\hline Toulouse & Occitanie & 1 & & 1500 \\
\hline Montreuil & Ile de France & 1 & \multirow{4}{*}{12} & 1500 \\
\hline Clamart & Ile de France & 1 & & 1500 \\
\hline Pontoise & Ile de France & 1 & & \\
\hline Paris & Ile de France & 2 & & 2000 \\
\hline
\end{tabular}

